\title{
Fine Structure of the Localized Emission from GaInNAs Layers Studied by Micro-Photoluminescence
}

\author{
R. Kudrawiec ${ }^{a, *}$, M. Latkowska ${ }^{a}$, G. SęK $^{a}$, J. Misiewicz $^{a}$, J. IbÁÑez ${ }^{b}$, M. Henini $^{c}$ \\ AND M. HOPKINSON ${ }^{d}$ \\ ${ }^{a}$ Institute of Physics, Wrocław University of Technology, Wybrzeże Wyspiańskiego 27, 50-370 Wrocław, Poland \\ ${ }^{b}$ Institut Jaume Almera, Consell Superior d'Investigacions Científiques, 08028 Barcelona, Spain \\ ${ }^{c}$ School of Physics and Astronomy, University of Nottingham, Nottingham NG7 2RD, United Kingdom \\ ${ }^{d}$ Department of Electronic and Electrical Engineering, University of Sheffield, S3 3JD Sheffield, United Kingdom

\begin{abstract}
GaInNAs bulk-like layers $(\approx 20 \%$ In and $\approx 3 \% \mathrm{~N})$ grown on GaAs substrate with various crystallographic orientations have been studied by micro-photoluminescence at low temperatures for a broad range of excitation conditions. In addition to photoluminescence peaks, which are associated with heavy- and light-hole free exciton recombination, a band of sharp lines was observed below the fundamental free exciton transition at low excitation. It shows that the localized emission which is typical of this alloy at low temperatures is composed of individual narrow photoluminescence lines which are associated with the recombination of single excitons. They can be localized on various local potential minima including those originating from the alloy content fluctuations and/or deep acceptor(donor)-like complexes.
\end{abstract}

PACS numbers: 78.55.Cr, 78.66.Fd

\section{Introduction}

GaInNAs alloy has received much attention in the past few years due to its unusual physical properties and promising applications in optoelectronic devices, especially GaAs-based lasers operating in the fiber optic telecommunications windows $(1.3$ and $1.55 \mu \mathrm{m})[1,2]$. The issue of material degradation with the increase in nitrogen content is one of the most important research aspects of this material system from the viewpoint of its application in diode lasers and solar cells. It is typical of this compound that the low temperature photoluminescence (PL) spectra measured at low excitation conditions are dominated by the recombination of localized carriers (excitons) trapped at local potential minima, where the exact nature of this recombination is still controversial. In general, it is expected that the broad PL band, which is attributed to the recombination of localized carriers (excitons), can be composed of sharp PL lines which are associated with individual excitons. An evidence for this scenario has been found in near-field photoluminescence spectroscopy [3-5] as well as in micro-photoluminescence $(\mu-\mathrm{PL})[6]$. In this work, we apply $\mu$-PL spectroscopy to study the energy fine structure of localized emission from GaInNAs bulk-like layers grown GaAs substrates with various crystallographic orientations.

* corresponding author; e-mail: robert.kudrawiec@pwr.wroc.pl

\section{Experimental}

$\mathrm{Ga}_{x} \operatorname{In}_{1-x} \mathrm{~N}_{y} \mathrm{As}_{1-y}$ thin films with $x \approx 20 \%$ and $y \approx 3 \%$ were deposited by molecular beam epitaxy (MBE) on epiready GaAs substrates with the following orientations: (100), (511)A, (411)A, (311)A, and (111)A. To grow the samples, a $0.2 \mu \mathrm{m}$ thick undoped GaAs buffer layer was first deposited at $T=590^{\circ} \mathrm{C}$ directly on the substrates. Then, the temperature was lowered to $T=400{ }^{\circ} \mathrm{C}$ and a $0.1 \mu \mathrm{m}$ thick undoped GaAs layer was grown, followed by the GaInNAs layer. The nominal thickness of the GaInNAs films was $100 \mathrm{~nm}$. Further relevant details on GaInNAs samples can be found in Ref. [7].

Micro-photoluminescence spectra were measured using a single grating $0.55 \mathrm{~m}$ focal length monochromator with a multichannel InGaAs detector. The $660 \mathrm{~nm}$ line of a semiconductor laser was focused on the sample by a high numerical aperture and long working distance microscope objective. The obtained diameter of the laser spot was estimated to be below $2 \mu \mathrm{m}$.

\section{Results and discussion}

Figure 1 shows low temperature $\mu$-PL spectra measured at various excitation conditions for GaInNAs layer (20\% In and $2.8 \% \mathrm{~N}$ ) grown on (311)A GaAs substrate. At low excitation power sharp individual lines are visible in the spectra. These lines showed no shift of spectral 
peak position with increasing excitation power however, the sharp features smeared out when the excitation power becomes large and the entire emission maximum shifts to blue apparently. A very similar behavior of $\mu$-PL spectra has previously been observed for GaNAs layers [6]. The smooth PL bands, which are dominant at high excitation, are associated with delocalized states in GaInNAs layer, namely, free exciton recombination, i.e. the heavy-hole $(\mathrm{HH})$ and light-hole ( $\mathrm{LH})$ exciton at lower and higher energy, respectively. The $\mathrm{HH}$ and $\mathrm{LH}$ transitions have been identified on the basis of reflectance and photoreflectance measurements at $10 \mathrm{~K}$. The sharp PL lines are separated from the $\mathrm{HH}$-transition by approximately $20 \mathrm{meV}$. Hence, they are attributed to localized states in this material where the micro-photoluminescence results provided an experimental evidence that this kind of localized recombination emission band in GaInNAs is composed of individual sharp lines each of which is associated with a single exciton localized at a characteristic potential minimum. These local minima are expected to have random properties and hence they should change with the position on the sample and show different thermal sensitivity. Therefore, we have performed position and temperature dependence of the photoluminescence.

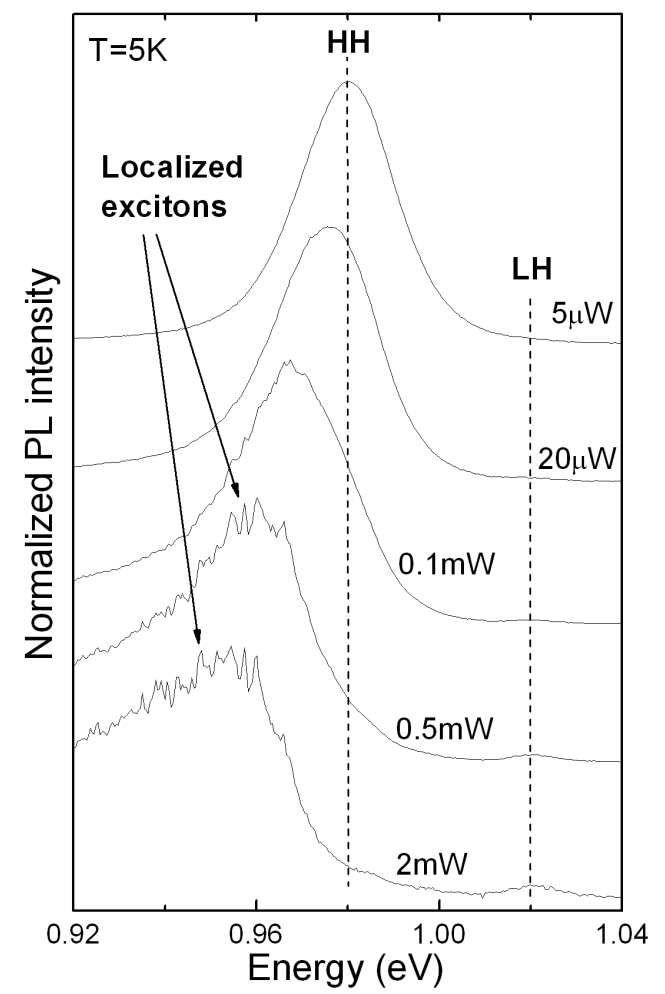

Fig. 1. Low temperature $\mu$-PL spectra measured at different excitation powers for GaInNAs layer grown on (311)A GaAs substrate.

Figure 2 shows $\mu$-PL spectra measured at different spots on the sample along a line with a step of $10 \mu \mathrm{m}$ with the excitation power of $10 \mu \mathrm{W}$. The energy posi- tions of the sharp lines vary with changing the excitation space. It is an evidence of the coexistence of very many localized excitons with various localization energies. Because of the spectral variation with the size of $10 \mu \mathrm{m}$, they cannot be resolved in a standard macro-PL spectra whose beam spot is typically several hundred $\mu \mathrm{m}$ and the spectrum is integrated over such an area. In order to visualize this effect, we present an imitated macro-PL spectrum in Fig. 2 (bold line) obtained from a simple summation of all the $\mu$-PL spectra plotted above.

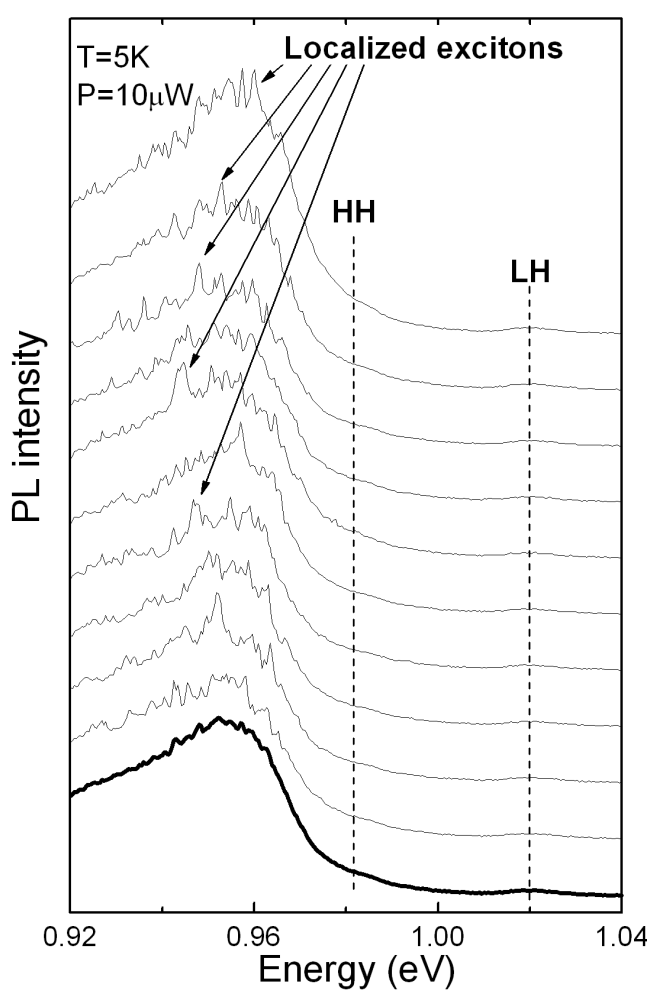

Fig. 2. Low temperature $\mu$-PL spectra measured along a line at the excitation power of $10 \mu \mathrm{W}$ for GaInNAs layer grown on (311)A GaAs substrate.

Figure 3 shows the temperature dependence of $\mu$-PL measured at the excitation power of $10 \mu \mathrm{W}$. This dependence is very analogous to the one which has been reported for GaNAs [6]. At low temperatures, the sharp lines are very well pronounced and they are observable at temperatures below $50 \mathrm{~K}$. Above this temperature, most of photogenerated carriers recombine non-radiatively. This result suggests the coexistence of radiative and non-radiative centers in this alloy. At low temperatures these radiative centers can be the origin of localized emission preventing the carriers to reach the possible non-radiative channels, whereas at higher temperatures when the carriers are thermally released and start traveling, the non-radiative centers may become active which leads to a dominant non-radiative recombination in the entire system. It is expected that deep acceptor- and donor-like states can play a role of both the 
radiative and non-radiative centers [6]. In addition to the sharp exciton lines associated with the deep acceptorand donor-like centers, alloy fluctuations can also lead to localized emission from GaInNAs. Such a scenario is often proposed for GaInNAs alloys due to the large miscibility gap in this alloy [4]. Finally, it is worth noting that sharp PL lines have been also observed for GaInNAs layers grown on other crystallographic orientations of GaAs substrate. As has been shown in Ref. [7] various crystallographic orientations influence on indium and nitrogen concentrations in GaInNAs layers as well as on their optical quality. Low temperature $\mu$-PL spectra are fingerprints of the optical quality and therefore they can be used to evaluate the quality of GaInNAs layers. Detailed comparison of $\mu$-PL spectra for GaInNAs layer grown on GaAs substrates with various crystallographic orientation will be presented elsewhere.

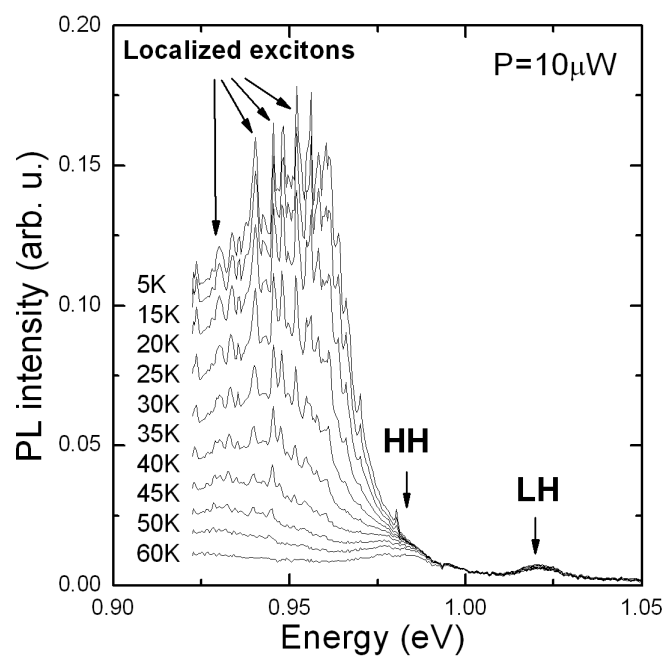

Fig. 3. Temperature dependence of $\mu$-PL spectra measured at the excitation power of $10 \mu \mathrm{W}$ for GaInNAs layer grown on (311)A GaAs substrate.

\section{Conclusion}

In conclusion, the energy fine structure of the localized emission from GaInNAs has been observed in $\mu$-PL spectra. It shows that the localized emission which is typical of dilute nitride alloys at low temperatures is composed of individual sharp PL lines which are associated with the recombination of individual excitons localized on various local potential minima. The temperature dependence of these sharp lines suggested that the deep acceptor and/or donor-like complexes can be the origin of the exciton localization at low temperatures.

\section{Acknowledgments}

R.K. acknowledges support from the Foundation for Polish Science and the MNiSW grant no. N N202 285634.

\section{References}

[1] M. Kondow, K. Uomi, A. Niwa, T. Kitatani, S. Watahiki, Y. Yazawa, Jpn. J. Appl. Phys. Part 1 35, 1273 (1996).

[2] M. Henini, Dilute Nitride Semiconductors, Elsevier, Oxford 2005.

[3] K. Matsuda, T. Saiki, M. Takahashi, A. Moto, S. Takagishi, Appl. Phys. Lett. 78, 1508 (2001).

[4] A.M. Mintairov, T.H. Kosel, J.L. Merz, P.A. Blabnov, A.S. Vlasov, V.M. Ustinov, R.E. Cook, Phys. Rev. Lett. 87, 277401 (2001).

[5] A.M. Mintairov, K. Sun, J.L. Merz, H. Yuen, S. Bank, M. Wistey, J.S. Harris, G. Peake, A. Egorov, V. Ustinov, R. Kudrawiec, J. Misiewicz, Semicond. Sci. Technol. 24, 075013 (2009).

[6] R. Kudrawiec, G. Sęk, J. Misiewicz, F. Ishikawa, A. Trampert, K.H. Ploog, Appl. Phys. Lett. 94, 011907 (2009).

[7] J. Ibandez, R. Kudrawiec, J. Misiewicz, M. Schmidbauer, M. Henini, M. Hopkinson, J. Appl. Phys. 100, 093522 (2006). 